\title{
Precios y oferta para el culantro castilla (Coriandrum sativum L.) entre los principales mercados mayoristas de Costa Rica y Panamá
}

Alexis Villalobos Monge'

Fecha de recepción: I de febrero del 201 I

Fecha de aprobación: 3 de setiembre del 2011 


\section{Palabras clave}

Culantro castilla, comercialización agrícola, precio, oferta.

\section{Resumen}

Este artículo muestra los resultados del análisis comparativo de precios y volúmenes ofertados del culantro de castilla en los principales mercados mayoristas de Costa Rica y Panamá. Como antecedente se tiene la ejecución de una gira de prospección realizada en el 2009 a Ciudad de Panamá por parte de los colaboradores del proyecto "Investigación de mercados agrícolas" de la Estación Experimental Fabio Baudrit de la Universidad de Costa Rica. En esta gira se detectó que el culantro castilla presentaba interesantes oportunidades de mercado gracias al precio y a la calidad del producto ofertado, razón por la cual se definió, como línea de trabajo, profundizar en aspectos de esta índole para determinar las particularidades de los principales mercados mayoristas de cada país en el caso de este alimento. Se llevó a cabo una revisión de series de tiempo para datos de precio y oferta emitidos por las fuentes encargadas de cada mercado, con los que se realizaron comparaciones de estadísticos y análisis de tendencia por regresión lineal, los cuales permitieron dimensionar las diferencias entre ambos sitios para enmarcar el comportamiento del culantro castilla como potencial agronegocio, relacionado con el consumo a nivel de cada país.

Entre los principales hallazgos, se puede mencionar que en Costa Rica históricamente ha existido un mayor consumo de esta hortaliza en comparación con Panamá, sin embargo, la tendencia detectada en Panamá, en cuanto a precios y volúmenes ofertados, está creciendo a mayor velocidad en este último mercado. Las diferencias promedio de precios pagados en Panamá superan por más del I 00\% a los pagados en Costa Rica por la misma unidad de comercialización según series mensuales para un periodo de 4 años, razón por la que se afinó la observación y se analizaron las plazas de comercialización para un total de 246 plazas mayoristas, donde se encontró que en el 94\% de las veces el precio fue mayor en el Mercado Central de Alimentos (MAC) en Panamá que en el Centro Nacional de Abastecimiento y Distribución de Alimentos (Cenada) en Costa Rica.

Se descubrió que las plazas con mayores diferencias de precios a favor del MAC se efectuaron entre la tercera semana de mayo y las tres primeras semanas de junio, por lo que se asume que serían los momentos más oportunos para obtener el mejor precio posible para ingresar al mercado panameño con culantro castilla costarricense.

\section{Key words}

Coriander, agricultural marketing, price, bid.

\begin{abstract}
This article shows the results of comparative analysis for prices and volumes offered for coriander in major wholesale markets in Costa Rica and Panama. As background there is the execution of an investigation job in 2009 to Panama City by the project partners "Research of markets for agricultural products" at Fabio Baudrit Experimental Station at the University of Costa Rica. During this job, it was found that coriander involves interesting market opportunities by the effects of price and quality of product offered, therefore it was defined as a line of work go deep into aspects of this kind to determine the particularities and characteristics of the major wholesale markets for this two countries in the case of this food. The methodology consisted of reviewing time series for price and supply data issued by the sources responsible for each market, which permit to make statistical comparisons and trend analysis by linear regression which allows measure the differences between the two sites for a better understanding of the behavior of coriander as a potential agribusiness, related to the consumption level of each country.
\end{abstract}

Among the main findings can be mentioned that Costa Rica has historically been a major consumer of this crop in comparison to what happened in Panama, but the trend detected in Panama in terms of prices and volumes offered is growing faster in the latter market. The average price difference of more than 100\% observed in Panama to comparison to Costa Rica for prices paid of the same marketing unit for a monthly series for a period of 4 years, hence the observation was refined and it was analyzed the squares marketing for a period at 246 squares wholesalers, it was found that in $94 \%$ of the times the price was higher in the Central Food Market (MAC) in Panama in comparison of the National Center for Food Supplies and Distribution (Cenada) in Costa Rica. It was found that the squares with higher price differences for the MAC occurred between the third week of May and the first three weeks of June, which means they would be more timely from the point of view of obtaining the best possible price to enter the Panamanian market with coriander from Costa Rica in these weeks. 


\section{Introducción}

La actualidad del sector hortícola costarricense es el resultado de una serie de experiencias productivas y comerciales desarrolladas históricamente por productores, profesionales y empresarios, quienes han contribuido de manera importante en la formación de una estructura comercial con un particular peso económico en muchos de los cantones que conforman el país.

Esta situación toma mayor relevancia cuando se observa el desarrollo que han alcanzado, tanto a nivel comercial como sociocultural, algunas de las agrocadenas hortícolas costarricenses, como es el caso de la papa en Cartago y el tomate en Alajuela y San José, solo para citar dos casos de hortalizas que ocupan sitios de preferencia dentro de los hábitos alimenticios de la población costarricense (PIMA, 2004). A pesar de la situación descrita, en los últimos años se ha puesto énfasis en la necesidad de considerar nuevas oportunidades de mercado para que el sector hortícola nacional continúe creciendo, tanto en oferta de productos como en mercados atendidos, razón por la que debe existir un esfuerzo permanente orientado a cumplir dicha premisa.

A partir de esto, los colaboradores del proyecto de investigación de mercados para productos agrícolas de la Estación Experimental Fabio Baudrit realizaron una gira de prospección al mercado panameño (Villalobos, 2009), la cual permitió determinar la existencia de varios productos hortícolas comercializados en Panamá con precios muy superiores a los pagados en Costa Rica y en condiciones de calidad por debajo de las acostumbradas por el consumidor nacional, por tal motivo, se definió como línea de trabajo la profundización en algunos detalles de mercado en pos de facilitar el entendimiento y el establecimiento de la dimensión real en virtud de la situación existente para tales productos.

Por lo tanto, en estas páginas se muestran los principales hallazgos y las conclusiones acerca de este producto que parece tener un interesante potencial de mercado: el culantro de castilla. Este alimento podría ajustarse a las condiciones del entorno, es decir, de los nuevos escenarios comerciales y a los consiguientes cambios en la oferta hortícola costarricense hacia mercados externos. Estos cambios, tentativamente beneficiosos, deben ser identificados con antelación y oportunamente si se va a poner en marcha una nueva propuesta de proyecto agrícola, lo cual se torna más plausible cuando la propuesta parte de un criterio técnico que demuestra la existencia de un grupo de personas que valorarían contar con una mejor alternativa del mismo producto/servicio que consumen, lo que en mercadeo se conoce como marketing de relaciones (Kotler, 2003).

En otras palabras, para asegurar la continuidad de los agronegocios hortícolas, tomando como base los fundamentos del marketing de relaciones, se vuelve imperativo identificar y entender la manera en que se debe atender la necesidad del cliente según el contexto comercial. Para el caso del culantro castilla y de los demás bienes y servicios en general, los Tratados de Libre Comercio (TLC) forman parte de los nuevos escenarios comerciales, donde identificar las oportunidades que estos instrumentos de política comercial puedan ofrecer. Particularmente, el más reciente tratado firmado por Costa Rica fue con la República de Panamá, de ahí que el trabajo que se expone se enfoca en este país.

En este trabajo se analizan aspectos de precios y oferta para el caso del culantro castilla (Coriandrum sativum L.) en los principales mercados mayoristas en Costa Rica y Panamá. Se espera que con este aporte se enriquezca la discusión sobre las oportunidades comerciales que tienen los productos hortícolas costarricenses en mercados internacionales.

\section{Materiales y métodos}

Este trabajo forma parte de las actividades del proyecto "Investigación de mercados para productos agrícolas", de la Estación Experimental Agrícola Fabio Baudrit Moreno (EEAFBM), e inscrito ante la Vicerrectoría de Investigación de la Universidad de Costa Rica (UCR).

Para su realización, inicialmente, se llevó a cabo la revisión de trabajos previos sobre el tema con el fin de comparar las estadísticas institucionales con los hallazgos hechos durante julio del 2009 en una gira realizada por investigadores del proyecto a Ciudad de Panamá.

Se analizaron estadísticas provenientes de los mercados mayoristas de alimentos frescos en ambos países. Se emplearon los boletines publicados por el Programa Integral de Mercadeo Agropecuario (PIMA) para el caso del Centro Nacional de 


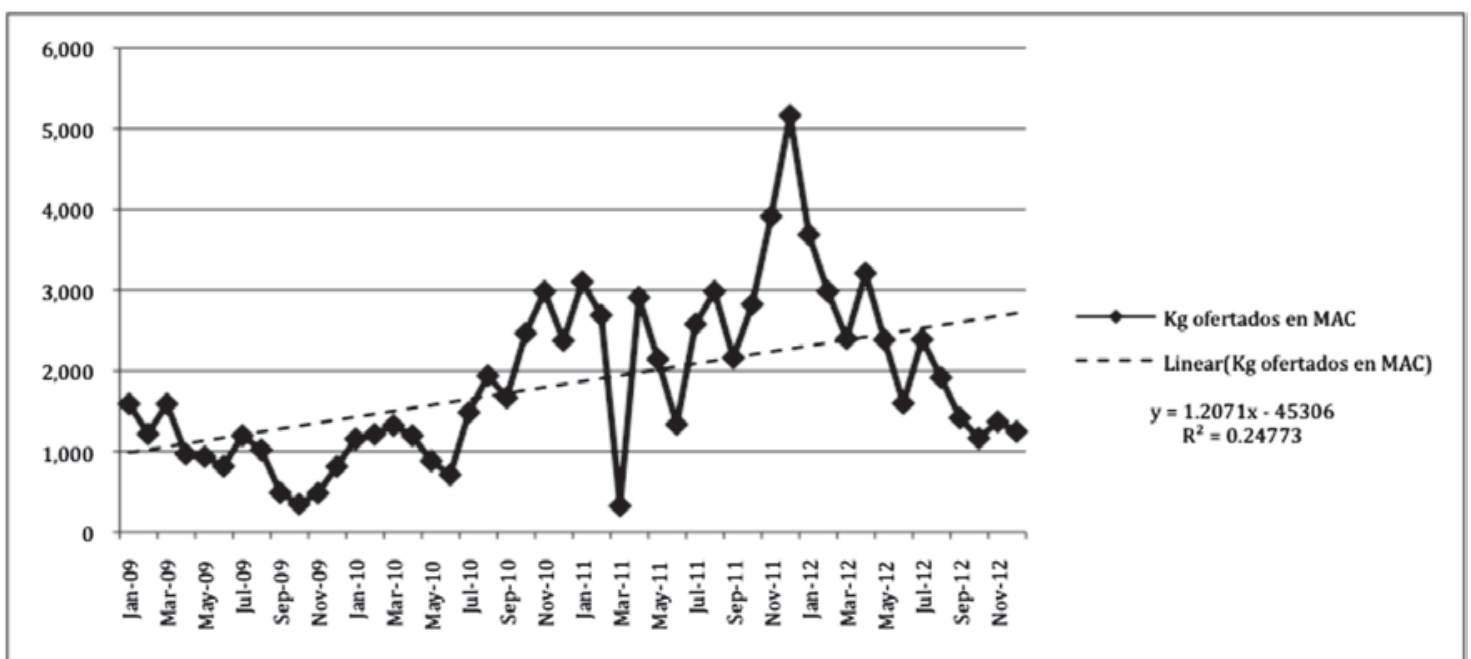

Gráfico I.Volúmenes promedio mensuales de oferta (en kg) de culantro castilla en el MAC. Periodo 2005-2008

Abastecimiento y Distribución de Alimentos (Cenada) en Costa Rica, así como los del Mercado Central de Alimentos (MAC) publicados por el Sistema para Agronegocios (SIPAN) del Instituto de Mercadeo Agropecuario (IMA) en Panamá.

El análisis comprende series de datos presentadas de dos formas:

- Valores mensuales: corresponden a los datos promedio por mes publicados por las instituciones mencionadas anteriormente, tanto para la variable "precio" como para la variable "oferta". Estos corresponden a un periodo de 48 meses entre enero del 2005 y diciembre del 2008.

- Valores por plaza de comercialización: son los datos del precio modal por plaza de comercialización recolectados de ambos países. Para el caso de los valores de precio en Panamá, la información fue enviada directamente por la fuente en dicho país y procesados por el autor. Se obtuvieron datos para el $58 \%$ de las plazas realizadas en Panamá en el periodo que va del 26 de enero del 2009 al 30 de agosto del 2010 ( I 44 plazas en total); mientras que se obtuvo información sobre el $100 \%$ de las plazas realizadas en el Cenada durante el mismo periodo (246 en total). En el caso del MAC, existe un $42 \%$ de valores perdidos por motivos internos de la fuente de información, los cuales salen del control del autor. Por tal motivo, el análisis se hace solo de las plazas de las que fue posible obtener la información para ambos mercados mayoristas.

Los datos provenientes de Panamá fueron estandarizados con los registros provenientes del PIMA con el propósito de realizar comparaciones con la misma base cuantitativa. Para esto se emplearon los siguientes criterios:

- Precios: los registros originales del MAC se encuentran en Balboas panameños, moneda que para el periodo en cuestión mantuvo una relación de paridad con el dólar estadounidense $(B / . I=\cup S \$ 1)$. Por tanto, la equiparación con el colón costarricense se realizó mediante el tipo de cambio de compra (TCC) para cada día específico de la realización de la plaza de comercialización, donde se empleó el registro que para tal efecto publica el Banco Central de Costa Rica (BCCR) en su sitio web.

- Oferta: además de la consideración del TCC, las unidades de comercialización son diferentes en ambos mercados, pues los boletines publicados del Cenada se refieren a "Rollos de 10 rollitos" (equivalentes en peso a valores aproximados de $450 \mathrm{~g})$ mientras que en el MAC se comercializa en "Mazos de $1 / 2$ libra" (equivalentes en peso a valores de $227 \mathrm{~g})^{2}$. Los valores equiparados se definieron en rollitos de $45 \mathrm{~g}$ cada uno, que es el 


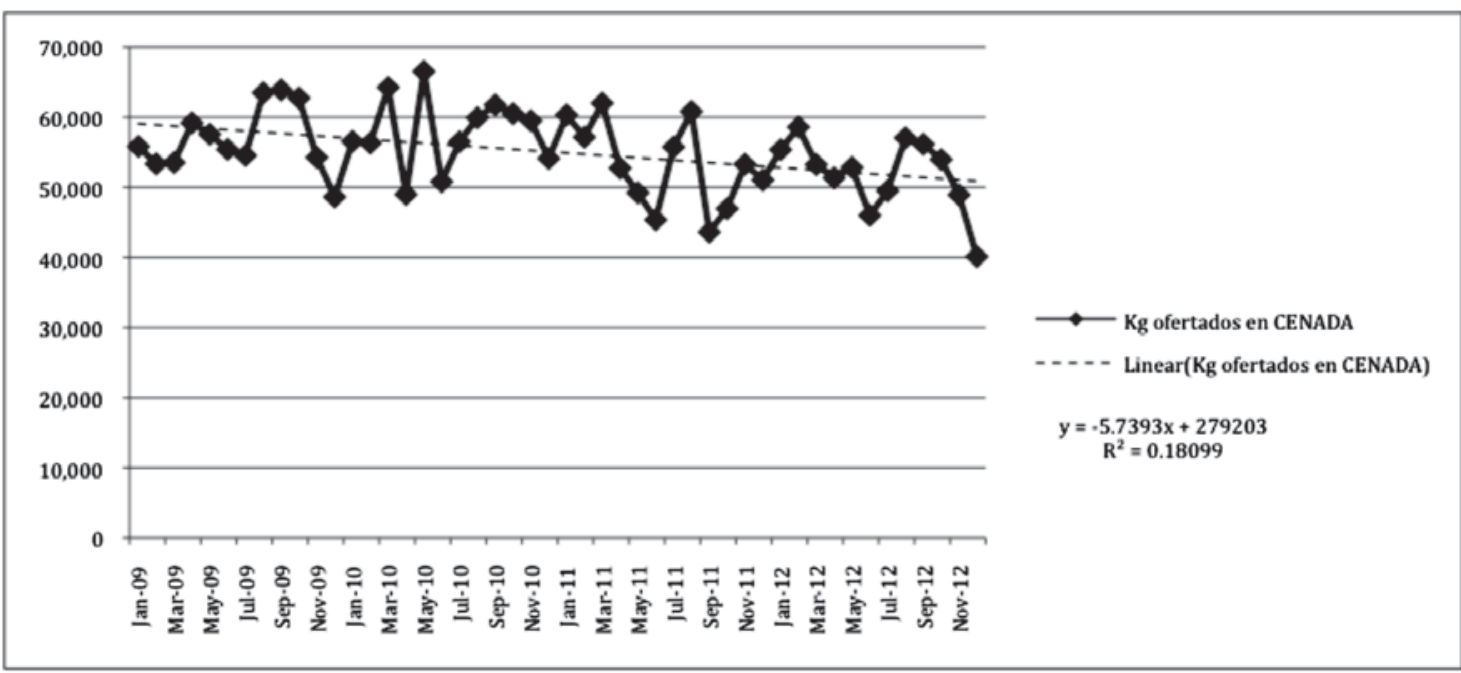

Gráfico 2.Volúmenes promedio mensuales de oferta (en kg) de culantro castilla en el Cenada. Periodo 2005-2008.

peso definido como parámetro por PIMA para efectos de comercialización mayorista.

- Nomenclatura: finalmente, se debió considerar el efecto cultural de la nomenclatura, esto porque el Coriandrum sativum L. comercializado en Costa Rica recibe el nombre de "Culantro de castilla", mientras que en Panamá recibe el nombre " $\mathrm{Ci}$ lantro chino". En los boletines del MAC, aparecen registros bajo el nombre "Culantro", el cual corresponde al "Culantro coyote" comercializado en Costa Rica según se constató en la gira de campo realizada en el 2009 a Ciudad Panamá.

Una vez conciliadas las diferencias anteriores, se procedió a tabular la información por fecha de plaza de comercialización en ambos mercados, y se comparó cada valor específico bajo la misma base monetaria en colones costarricenses (para el caso de la variable "precio") y de peso en kilogramos (para la variable "volumen ofertado").

El procesamiento de la información se realizó con el programa Excel para la construcción de gráficos y regresiones lineales así como el programa estadístico Statistical Package for Social Sciences versión 15.0 (SPSS por sus siglas en inglés) para el manejo de las series de datos en cuanto a la obtención de valores de tendencia central y variabilidad, así como coeficientes de correlación entre las diferentes series.

\section{Resultados y discusión}

Análisis de los volúmenes mensuales ofertados en ambos mercados mayoristas

El análisis consideró el manejo de datos en series mensuales por cada mercado mayorista, y se establecieron comportamientos y tendencias durante los periodos considerados. Para el caso de la variable "oferta" de culantro castilla en el MAC, fue posible construir gráficos que sintetizan la información tabular compilada, como se puede ver en el gráfico I.

Los resultados obtenidos muestran que, en el periodo en cuestión, la oferta del culantro castilla mantuvo un comportamiento con tendencia al alza (valor positivo de la pendiente de ajuste de 1,23). Asimismo, se observa un "pico" de oferta para el mes de diciembre del 2007 con un valor de $5.166 \mathrm{~kg}$, con un promedio de $1.91 \mathrm{I} \pm 1.028 \mathrm{~kg}$ al mes.

Para el caso del Cenada, los valores compilados muestran una tendencia diferente a la observada en el MAC (gráfico 2).

Para el Cenada, se detectó una tendencia negativa en la oferta (explicada por la pendiente de -5,7 de la ecuación de ajuste lineal), con un promedio mensual de oferta de $54.991 \pm 5.749 \mathrm{~kg}$ al mes y un pico de oferta en mayo del 2006 de 66.540 kg. En los cuadros 1 y 2 se muestran las estadísticas descriptivas para ambos grupos de datos. 
Cuadro I. Estadísticas de la oferta en el MAC.

\begin{tabular}{lr}
\hline \multicolumn{2}{c}{ Kg ofertados en MAC } \\
\hline & \\
Media & 1910,56818 \\
Error típico & 148,400889 \\
Mediana & \multicolumn{1}{c}{ 1594,09091 } \\
Moda & \multicolumn{1}{c}{ 1028, A } \\
Desviación estándar & \multicolumn{1}{l}{15152} \\
Varianza de la muestra & $0,67095,55$ \\
Curto sis & 0,64662527 \\
Co eficiente de asimetría & 0,82742056 \\
Rango & 4817,72727 \\
Mínimo & 348,181818 \\
Máximo & 5165,90909 \\
Suma & 91707,2727 \\
Cuenta & 48 \\
Nivel de confianza(95,0\%) & 298,544077 \\
\hline
\end{tabular}

Cuadro 2. Estadísticas de la oferta en el Cenada.

\begin{tabular}{lr}
\hline \multicolumn{2}{c}{$K g$ ofertados en CENADA } \\
\hline & \\
Media & 54990,625 \\
Error típico & 829,758435 \\
Mediana & 55390 \\
Moda & \#N/A \\
Desviación estándar & 5748,73507 \\
Varianza de la muestra & 33047954,9 \\
Curto sis & $-0,07585859$ \\
Co eficiente de asimetría & $-0,29275347$ \\
Rango & 26420 \\
Mínimo & 40120 \\
Máximo & 66540 \\
Suma & 2639550 \\
Cuenta & 48 \\
Nivel de confianza(95,0\%) & 1669,25863 \\
\hline
\end{tabular}

Fuente: Elaboración propia con datos de PIMA y SIPAN, 2010.

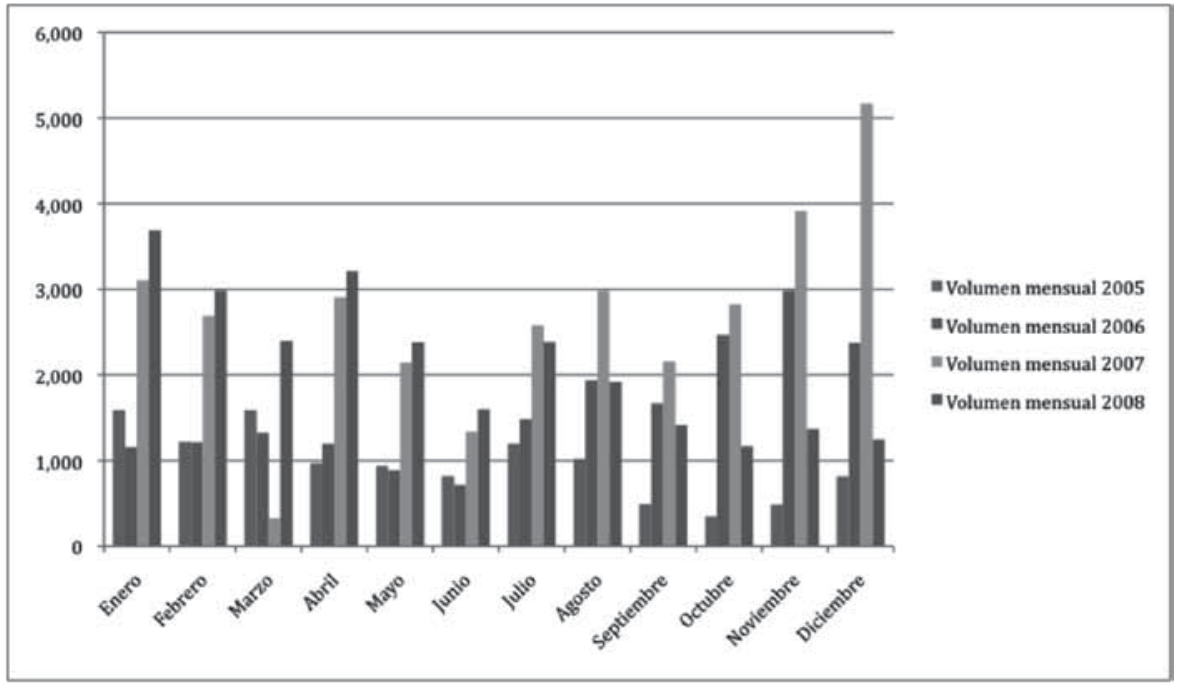

Gráfico 3. Volúmenes ofertados por mes (en kg) registrados en el MAC. Periodo 2005-2008.

Debe destacarse que las medias de oferta tienen diferencias claramente marcadas, ya que los volúmenes comercializados en Cenada son un 2,878\% mayor a los transados en el MAC durante el periodo en cuestión. También hay que apuntar que la variabilidad de la oferta fue menor en el Cenada, pues el coeficiente de variación fue de un 10,45\% durante el periodo, mientras que para el caso del MAC fue de un $53,81 \%$. Ambos estadísticos suponen una oferta con una mayor estabilidad en cuanto al volumen mensual esperable para el caso del Cenada en comparación al caso del MAC en Panamá.
La correlación que existe entre ambas variables es levemente negativa, debido al valor $-0,08$ obtenido del coeficiente de correlación de Pearson. Esto sugiere la existencia de una débil relación, la cual supone que ante aumentos en la oferta de culantro castilla en el Cenada se esperaría una leve disminución en los volúmenes transados en el MAC; sin embargo, este estadístico no es concluyente, pues se puede establecer la independencia lineal entre ambas variables, según lo sugiere Devore (2008), en el caso de presentarse este tipo de resultados para dicho estadístico. 


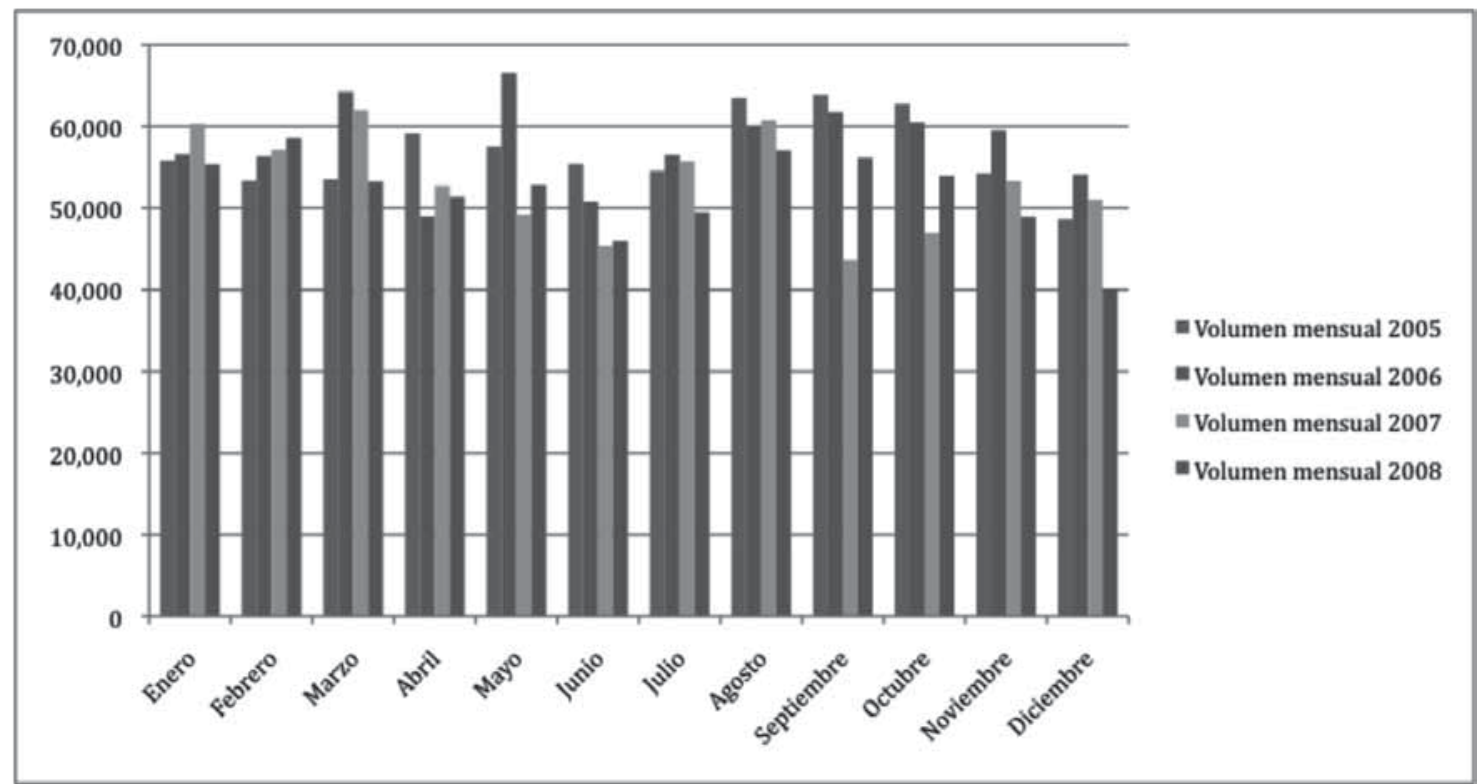

Gráfico 4.Volúmenes ofertados por mes (en kg) registrados en el Cenada. Periodo 2005-2008.

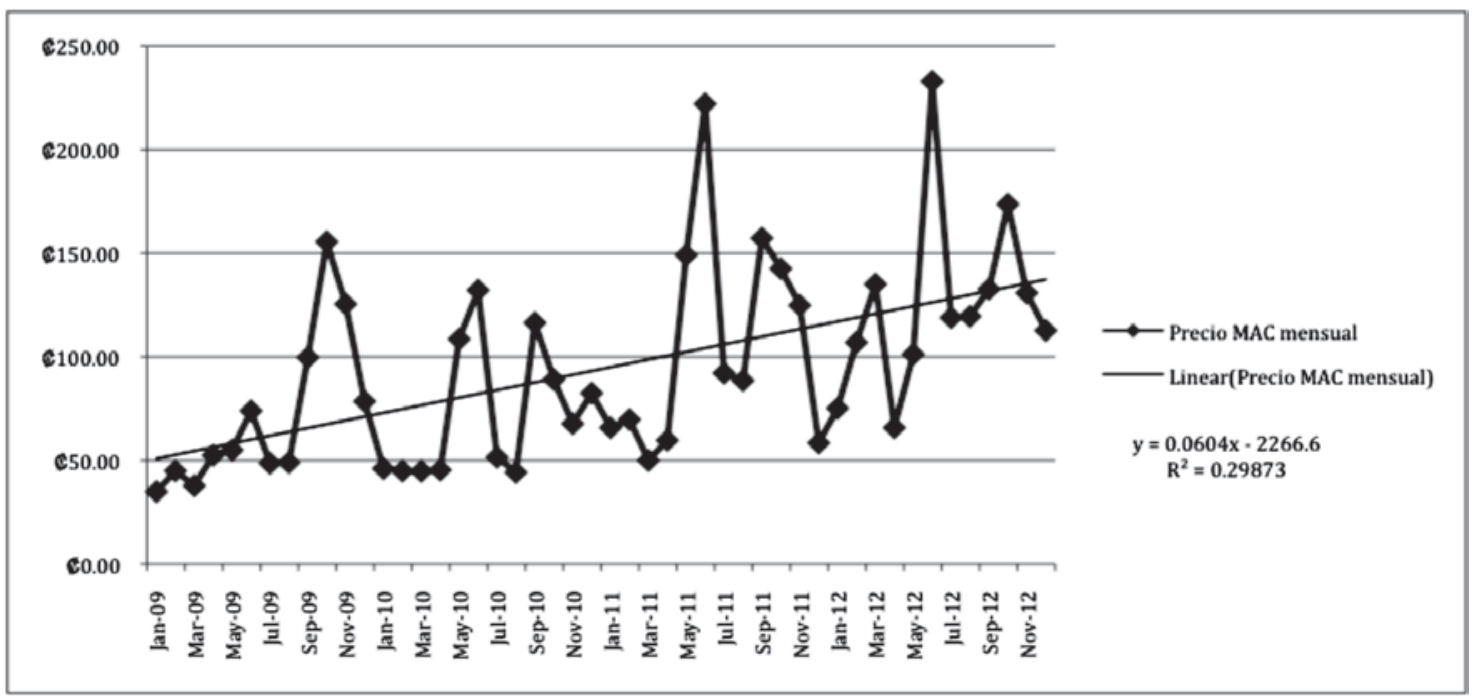

Gráfico 5. Precios promedio por mes (en colones por rollito) registrados en el MAC. Periodo 2005-2008.

Al comparar el comportamiento de los volúmenes ofertados en ambos mercados, se destaca la existencia de algunos meses con valores mínimos y máximos a lo largo de las series analizadas, principalmente en el MAC, porque en el mes de junio de cada año se observan, de manera consistente, valores bajos en comparación a los restantes meses, mien- tras que para el caso de aquellos meses con valores máximos parece no presentarse un comportamiento claramente definido (gráfico 3).

En el caso del Cenada, no se observa un mes que consistentemente presente un valor mínimo de oferta o un valor máximo dentro de la serie 


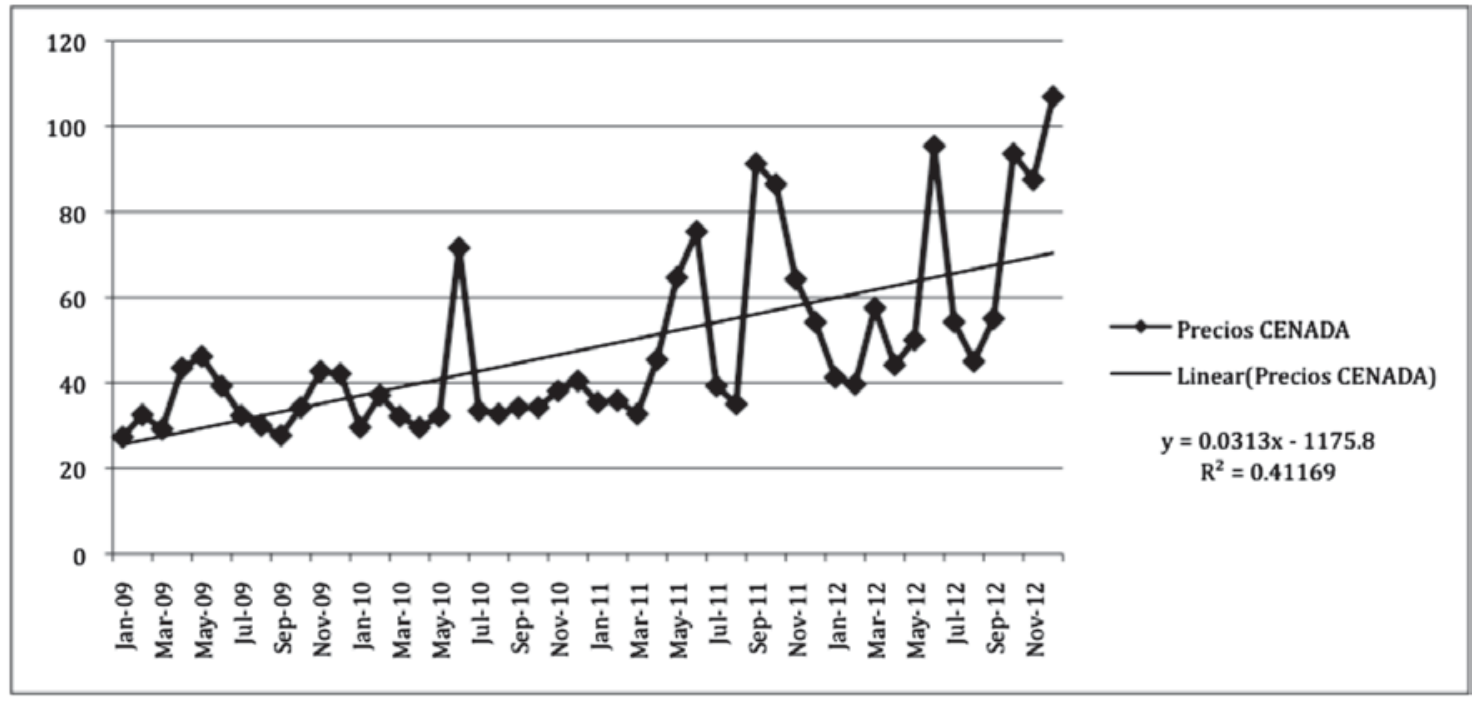

Gráfico 6. Precios promedio por mes (en colones por rollito) registrados en el Cenada. Periodo 2005-2008.

Cuadro 3. Estadísticas del precio en el MAC.

\begin{tabular}{lr}
\hline \multicolumn{2}{c}{ Precios en el MAC } \\
\hline & \\
Media & 94,0357144 \\
Error típico & 6,8000601 \\
Mediana & \multicolumn{1}{c}{ 85,493176 } \\
Moda & \multicolumn{1}{c}{ \#N/A } \\
Desviación estándar & \multicolumn{1}{l}{ 47,1121984 } \\
Varianza de la muestra & 2219,55923 \\
Curto sis & 0,82486168 \\
Co eficiente de asimetría & 0,98480438 \\
Rango & 197,998005 \\
Mínimo & 34,9016136 \\
Máximo & 232,899618 \\
Suma & 4513,71429 \\
Cuenta & 48 \\
Nivel de confianza(95,0\%) & 13,6799562 \\
\hline
\end{tabular}

Cuadro 4. Estadísticas del precio en el Cenada

\begin{tabular}{lr}
\hline \multicolumn{2}{c}{ Precios en el CENADA } \\
\hline & \\
Media & 47,953125 \\
Error típico & 3,00271569 \\
Mediana & 40,016 \\
Moda & 34,231 \\
Desviación estándar & 20,8034246 \\
Varianza de la muestra & 432,782474 \\
Curto sis & 0,94223946 \\
Coeficiente de asimetría & 1,39186222 \\
Rango & 79,615 \\
Mínimo & 27,308 \\
Máximo & 106,923 \\
Suma & 2301,75 \\
Cuenta & 48 \\
Nivel de confianza(95,0\%) & 6,04068471 \\
\hline
\end{tabular}

Fuente: Elaboración propia con datos de PIMA y SIPAN, 2010.

considerada, aunque se da el caso del mes de diciembre, que presenta valores mínimos para 2 de los 4 periodos analizados (gráfico 4).

Análisis de los precios mensuales entre ambos mercados mayoristas

En cuanto al tratamiento de la variable "precio", para el caso del MAC (Mercado, 2009) se encontraron los valores promedio por mes que se muestran en el gráfico 5.

En el gráfico se observa una tendencia al alza para esta variable, con un precio promedio para dicho periodo de $95 \pm 47$ colones por rollito.

Para el caso del Cenada (PIMA, 2009), también se observa una tendencia hacia el alza para esta variable, con valores promedio de $48 \pm 21$ colones por 


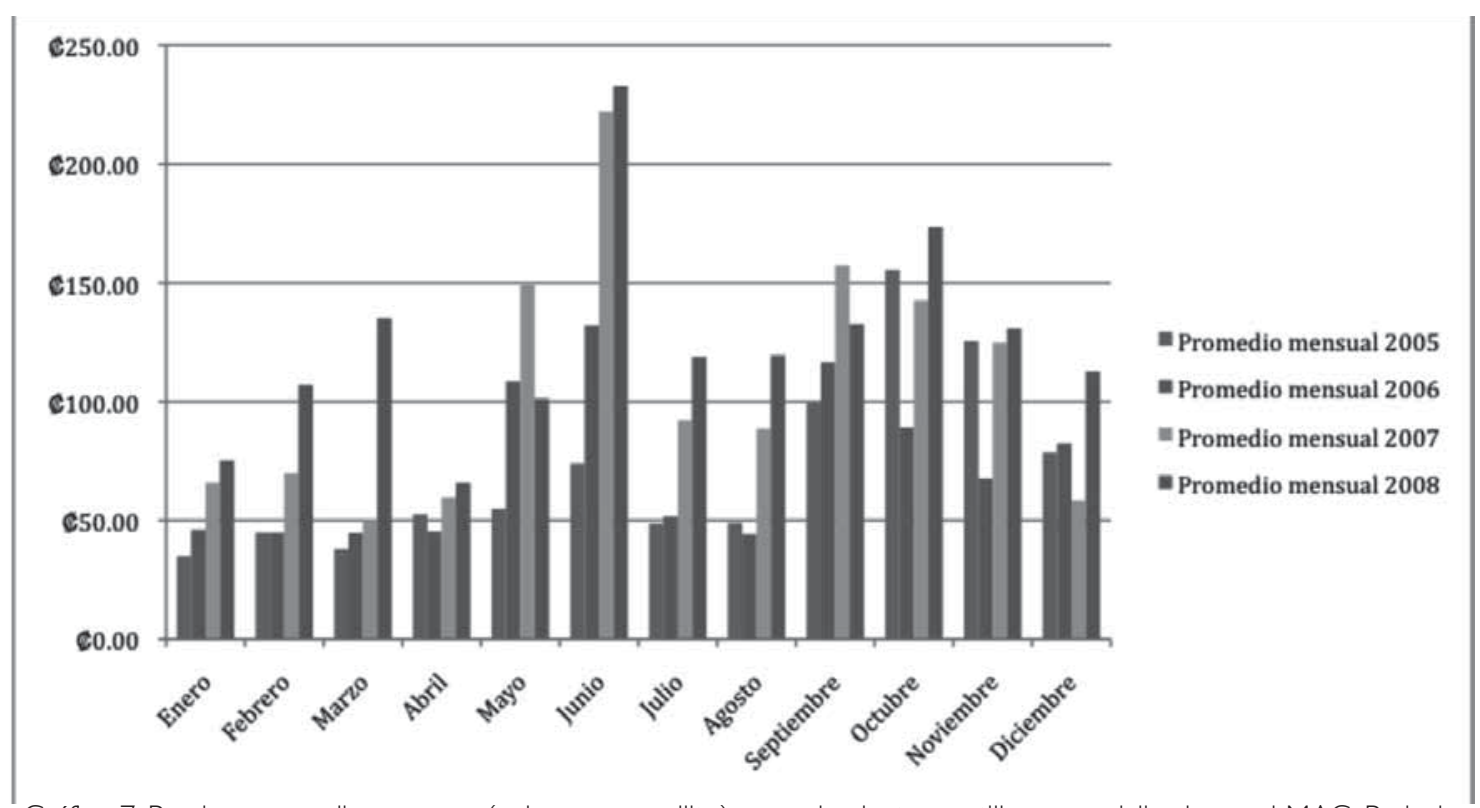

Gráfico 7. Precios promedio por mes (colones por rollito) para el culantro castilla comercializado en el MAC. Periodo 2005-2008.

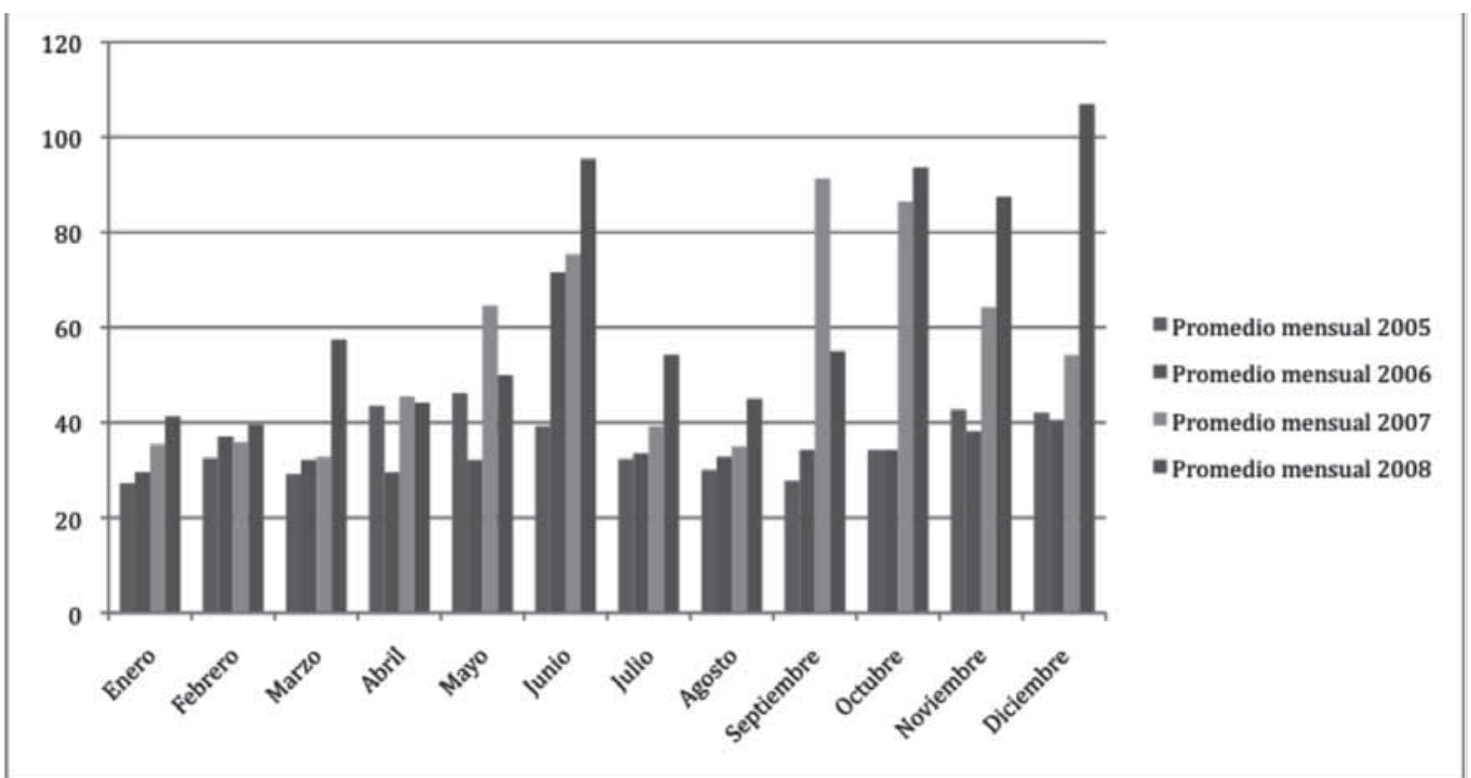

Gráfico 8. Precios promedio por mes (colones por rollito) para el culantro castilla comercializado en el Cenada. Periodo 2005-2008.

rollito. Entre ambos mercados, el MAC presenta una pendiente mayor para el caso del aumento del precio $(0,06)$, lo cual supone una expectativa de que el ritmo en que el precio aumentará en el futuro.

En los gráficos 3 y 4 se observan las estadísticas descriptivas para ambas series de datos: la media del precio promedio en el Cenada representa el 5I\% del valor medio encontrado en el MAC. La variabilidad expresada por el coeficiente de variación es similar entre ambos mercados durante el periodo descrito, puesto que en el MAC fue de un $50,1 \%$ y en el Cenada fue de un 43,4\%. El rango observado 


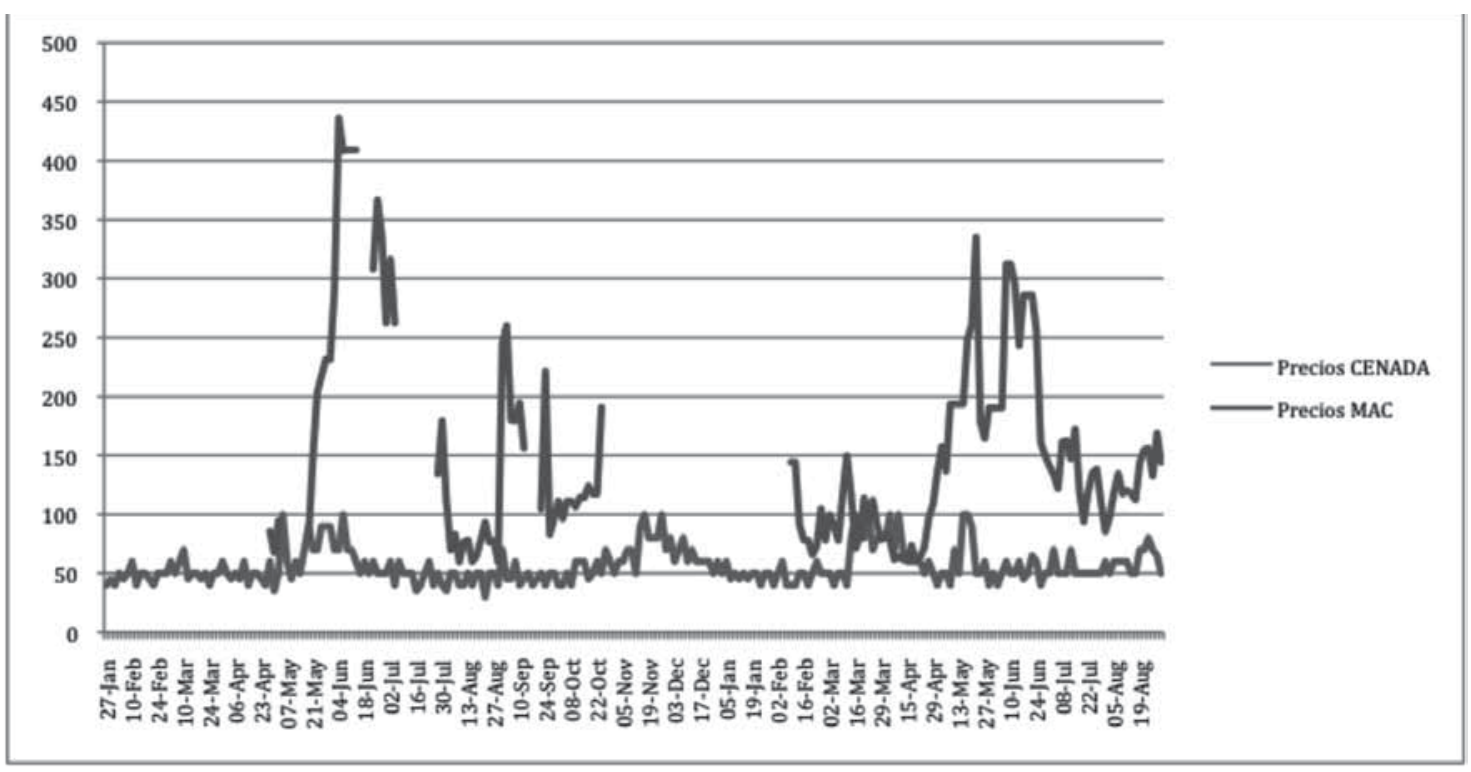

Gráfico 9. Comparación por plaza (en colones por rollito) para el precio del culantro castilla en el MAC y en el Cenada. Periodo 2009-2010.'

I. Los vacíos o valores perdidos de información para el caso del MAC son responsabilidad de la fuente emisora de la información.

en el MAC fue mayor ( $\not 198$ por rollito) en comparación al Cenada ( $\not 80$ por rollito), razón por la que resulta importante analizar el efecto de plaza de comercialización para encontrar el momento en que se presenten ventanas puntuales en que los precios sean mayores en el mercado panameño, comparado con el Cenada. Lo anterior se refuerza al observar que el coeficiente de correlación obtenido fue de 0,73 , lo cual muestra una fuerte relación lineal entre las variables para ambos mercados; esto supone que cuando el precio aumenta en un mercado, en el 73\% de las veces también lo hará en el otro.

En el gráfico 7 se muestran los valores promedio para el precio en el MAC, del que se desprende que los meses de junio, particularmente (con valores de ф233 por rollito en el 2008), así como setiembre y octubre presentan los mayores precios en promedio, mientras que los menores precios en promedio se observan en los meses de abril principalmente (con valores de $\$ 66$ por rollito en el 2008), así como enero y febrero.

Para el caso del Cenada, se observa que los meses de junio, setiembre, octubre y diciembre presentan los mayores niveles de precios para el periodo en cuestión (el mes de diciembre del 2008 superó la barrera de los $\not 100$ por rollito, con un valor de $\not 1$ 07). Los meses con menores precios en promedio corresponden al periodo de enero a abril, ya que para el 2008 el mes de febrero presentó precios promedio de $\$ 39$ por rollito. El detalle se resume en el gráfico 8.

Análisis de los precios por plaza de comercialización entre ambos mercados mayoristas

Para detectar el efecto que la variación de los precios por plaza particular tiene en el cálculo de los precios promedio por mes, se realizó una comparación para el periodo de enero del 2009 a octubre del 2010. Este ejercicio permitió determinar que para el mes de junio del 2009 y 2010 , pese a la existencia de un valor promedio de $\$ 363$ y $ф 239$ por rollito, respectivamente, se presentaron plazas con precios superiores en un 20\% para el 2009 y en un 31\% para el 2010 en el MAC. El gráfico 9 muestra los valores por plaza con el fin de validar este hallazgo.

Es posible observar que, con la información disponible de 276 plazas en el Cenada y I 44 en el MAC, se puede afirmar que los precios para el culantro castilla en Panamá son superiores a los registrados 


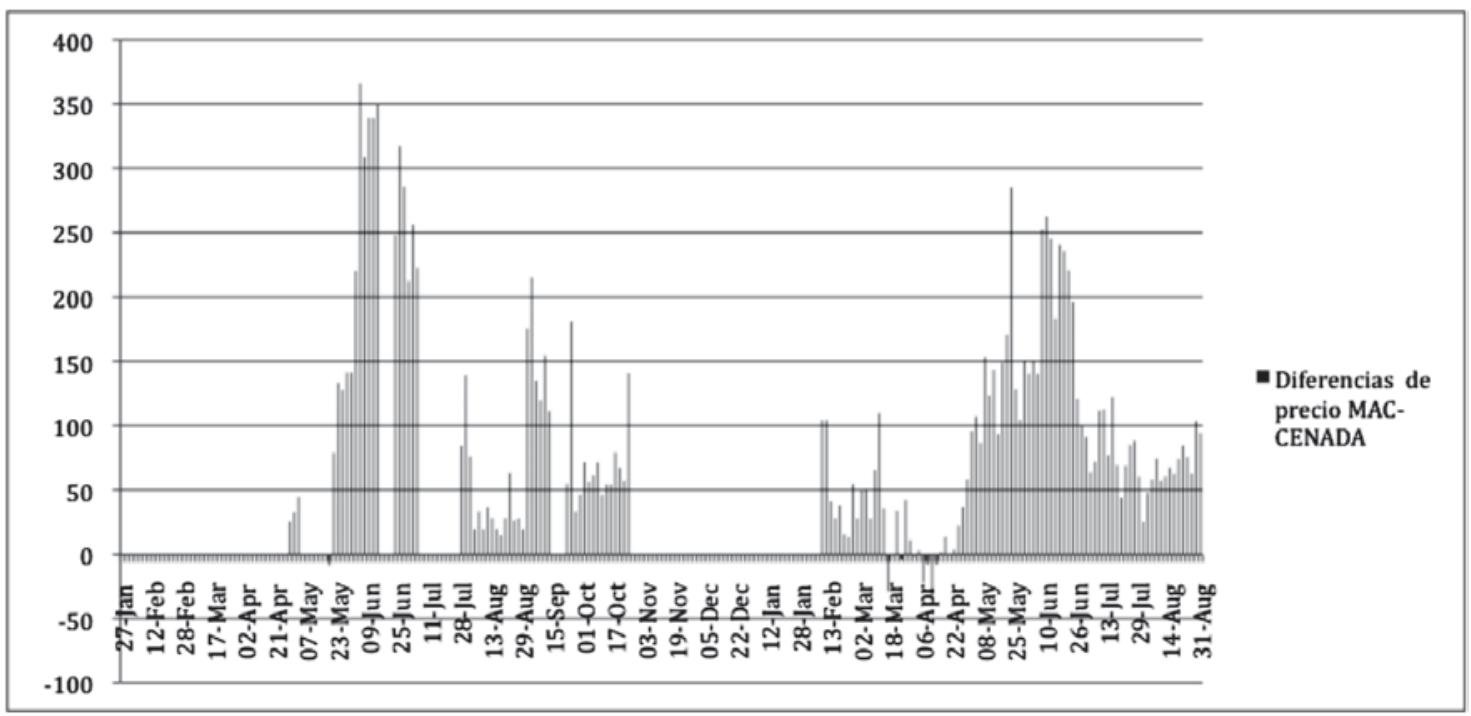

Gráfico 10. Comparación de las diferencias absolutas encontradas por plaza (en colones por rollito) para el precio del culantro castilla en el MAC y en el Cenada. Periodo 2009-20I0.

en el principal mercado mayorista de Costa Rica en el $94,5 \%$ de las plazas con registros observados, en especial durante los meses con registros promedios superiores (junio, setiembre y octubre).

Las mayores diferencias se observan a partir de las plazas realizadas de la cuarta semana de mayo, con diferencias superiores a los $\not \mid 50$ por rollito a favor del MAC, hasta la tercera y cuarta semana de junio, un periodo en el que se efectuaron entre 14 y 17 plazas de comercialización en el MAC (gráfico 10).

Las diferencias detectadas, superiores a los $\$ 350$ por rollito comercializado en algunas plazas del periodo descrito, significa, en términos relativos, variaciones de un 523\% en el precio existente en el Cenada para la misma fecha de la plaza. Las diferencias promedio que se observan entre el periodo descrito, a partir de la tercer semana de mayo hasta finales de junio, corresponden al 370,5\% para el 2009, y del $356,4 \%$ para el 2010 , donde este lapso de plazas de comercialización mostró, durante el periodo de análisis, las mayores diferencias tanto en términos relativos como en términos absolutos. Para futuros esfuerzos de comercialización, esta "ventana" de precios podría considerarse como el mejor momento para realizar dicho esfuerzo, sin embargo, se debe reconocer que los valores perdidos para el caso del MAC podrían representar un factor de error si se considera que estas plazas son efectivamente las que presentaron las mayores diferencias de precios entre ambos mercados mayoristas.

\section{Conclusiones}

Los resultados que se han encontrado hasta la fecha permiten establecer algunas consideraciones relevantes.

En primer lugar, el comportamiento de la oferta de culantro castilla mantiene una tendencia hacia el alza en ambos mercados, con una mayor intensidad para el caso del MAC al compararse con el Cenada. Esto resulta interesante dado el hecho de que esta hortaliza de hoja no es la principal "aromática" de consumo por el panameño, sino que es el "culantro coyote" el que se consume con mayor frecuencia, e inclusive se le conoce solo como "culantro" en ese país. Sin embargo, es notorio cómo ha aumentado la oferta de esta hortaliza (culantro castilla) en el MAC, al pasar de volúmenes cercanos a los $350 \mathrm{~kg}$ (octubre del 2005) hasta más de $5000 \mathrm{~kg}$ (diciembre del 2007) mensuales.

Al contrastar estos resultados con la oferta registrada en el Cenada, se observa que también existe un comportamiento hacia el alza, donde se evidencia que el consumo de esta hortaliza es mayor en 
Costa Rica (por los volúmenes comercializados) en comparación a lo que sucede actualmente en Panamá, donde se han presentado volúmenes ofertados, como el observado en el mes de mayo del 2006, con un registro de $66.540 \mathrm{~kg}$. Los estadísticos obtenidos para ambas series apoyan estas aseveraciones ya que los estimadores de variabilidad suponen una oferta más estable en el Cenada, quizás debido a que el consumo de esta hortaliza es más común en Costa Rica, lo que deja para trabajos complementarios la evaluación del crecimiento potencial que la población panameña podría tener por el consumo de culantro castilla en el futuro.

También se observa que en ambos mercados existen meses con marcados aumentos y disminuciones en la oferta de este alimento, por lo que habría que evaluar en el futuro si esta situación obedece a efectos culturales, climatológicos o a una combinación de estas y otras variables.

En segundo lugar, los precios medidos en la misma unidad de comercialización (rollito) permiten establecer que en Panamá se registran precios muy superiores a los que se pagan en Costa Rica por esta hortaliza de hoja. Los precios promedio por mes establecen valores históricos de $\not 48 /$ rollito en el Cenada y $\not 1105 /$ rollito en el MAC, una diferencia 2,2 veces en favor del mercado mayorista panameño. También en este mercado existe mayor variabilidad para el precio en comparación con el Cenada (medido por un valor para el Coeficiente de Variación de 56\% para el MAC y $43 \%$ para el Cenada), sin embargo, las diferencias no son tan marcadas como ocurre para el caso de la variable "volumen ofertado".

Este último aspecto lleva a una tercera consideración: al parecer existe una ventana de mercado establecida en un lapso de tiempo definido, entre la última semana de mayo y las cuatro semanas de junio, puesto que, a pesar de verificar que el precio en el MAC normalmente fue mayor en 136 de las I 44 plazas con información disponible (94,4\% del total), fue en estas semanas en que se alcanzó, tanto en las plazas registradas en el 2009 como en el 2010 , las mayores diferencias con respecto a los precios registrados en el Cenada, como se constató en el gráfico I0. Este hecho es relevante en el sentido de que se identificó un periodo en el tiempo en que se podrían minimizar los riesgos de encontrar bajos precios para potenciales esfuerzos de exportación de este alimento hacia el mercado panameño, donde las semanas indicadas podrían considerarse los mejores momentos durante el año si se quiere obtener las mayores retribuciones monetarias posibles.

\section{Bibliografía}

Devore, J., (2008). Probabilidad y estadística para ingeniería y ciencias. México: Cengage Learning.

Kotler, P. (2003). Fundamentos de marketing. Madrid: Prentice-Hall.

Mercado, D.d. (2009). Boletín semanal de precios en el MAC. Panamá.

Programa Integral de Mercadeo Agropecuario [PIMA]. (2004). Tendencias del consumo de frutas, hortalizas y pescado en las familias de Costa Rica.

Programa Integral de Mercadeo Agropecuario [PIMA]. (2009). Boletines de precios de venta del mayorista al minorista en el Cenada. Recuperado de http:// www.pima.go.cr/Controls.aspx?control=ViewBo_ asc $\times \& e n t i d a d=$ Cenada.

Programa Integral de Mercadeo Agropecuario [PIMA]. (20 I0). Manual de consultas e índices estacionales de los principales productos hortifrutícolas que se comercializan en el Cenada.

Sistema de Agronegocios [SIPAN]. (20I0) Estadísticas de precios y oferta presentado en el MAC. Recuperado de http://www.ima.gob.pa/ima/sipanpagedetail. aspx?.pcode_Info3698\&spcode=Naci2368

Villalobos, A. (2009). Informe 2009 del Proyecto 736-A7059: Investigación de mercados para productos agrícolas. Informe parcial. 
Tecnología en Marcha,

44 Vol. 25, N. I, Enero-Marzo 2012 